Check for updates

Cite this: Phys. Chem. Chem. Phys., 2018, 20, 17714

Received 14th April 2018 , Accepted 30th May 2018 DOI: $10.1039 / c 8 c p 02397 b$ rsc.li/pccp

\title{
Effects of probe energy and competing pathways on time-resolved photoelectron spectroscopy: the ring-opening of 1,3-cyclohexadiene
}

\author{
Maria Tudorovskaya, ${ }^{a}$ Russell S. Minns ${ }^{b}$ and Adam Kirrander (D) *a
}

\begin{abstract}
The ring-opening dynamics of 1,3-cyclohexadiene (CHD) following UV excitation is studied using a model based on quantum molecular dynamics simulations with the ab initio multiconfigurational Ehrenfest (AI-MCE) method coupled to the Dyson orbital approach for photoionisation cross sections. Time-dependent photoelectron spectra are calculated for probe photon energies in the range 2-15 eV. The calculations demonstrate the value of universal high-energy probes, capable of tracking the dynamics in full, but also the utility of more selective lower-energy probes with a more restricted ionisation range. The predicted signal, especially with the universal probes, becomes highly convoluted due to the contributions from multiple reaction paths, rendering interpretation challenging unless complementary measurements and theoretical comparisons are available.
\end{abstract}

\section{Introduction}

The molecule 1,3-cyclohexadiene (CHD) is a six-membered carbon ring that undergoes an ultrafast ring-opening reaction following excitation by UV light. The reaction forms an essential part of the biosynthesis of Previtamin D3, the thermal precursor to the human nutrient Vitamin $\mathrm{D} 3,{ }^{1-3}$ and is involved in a number of interesting applications including photochromic dyes $^{4,5}$ and devices, ${ }^{6}$ optical switching, ${ }^{7}$ and nanomechanical motors. ${ }^{8}$ In broad terms, the reaction is understood by the mechanism schematically outlined in Fig. 1. ${ }^{9-11}$ Absorption of UV light at approximately $266 \mathrm{~nm}$ places the wavepacket on the steeply repulsive potential energy surface of the optically bright 1B ${ }^{1} \pi \pi^{*}$ state, with the wavepacket accelerated ballistically towards the diabatic $2 \mathrm{~A}$ spectroscopically dark state on a $<50 \mathrm{fs}$ timescale. It returns to the $1 \mathrm{~A}$ electronic ground state through a conical intersection, leading to either the ring-open 1,3,5hexatriene (HT) photoproduct or the ring-closed CHD molecule. The main steps in the reaction are concluded within $200 \mathrm{fs}$, providing a spectacular example of fast internal conversion. The molecule carries a large excess of vibrational energy, once it has relaxed back to the electronic ground state. In solution, this energy is dissipated to the bath, cooling the internal temperature from an estimated $2200 \mathrm{~K}$ to the ambient temperature of the surroundings. ${ }^{12}$

\footnotetext{
${ }^{a}$ EaStCHEM, School of Chemistry, University of Edinburgh, David Brewster Road, EH9 3FJ, Edinburgh, UK. E-mail: Adam.Kirrander@ed.ac.uk; Tel: $+44(0) 1316504716$

${ }^{b}$ Chemistry, University of Southampton, Highfield, SO17 1BJ, Southampton, UK
}

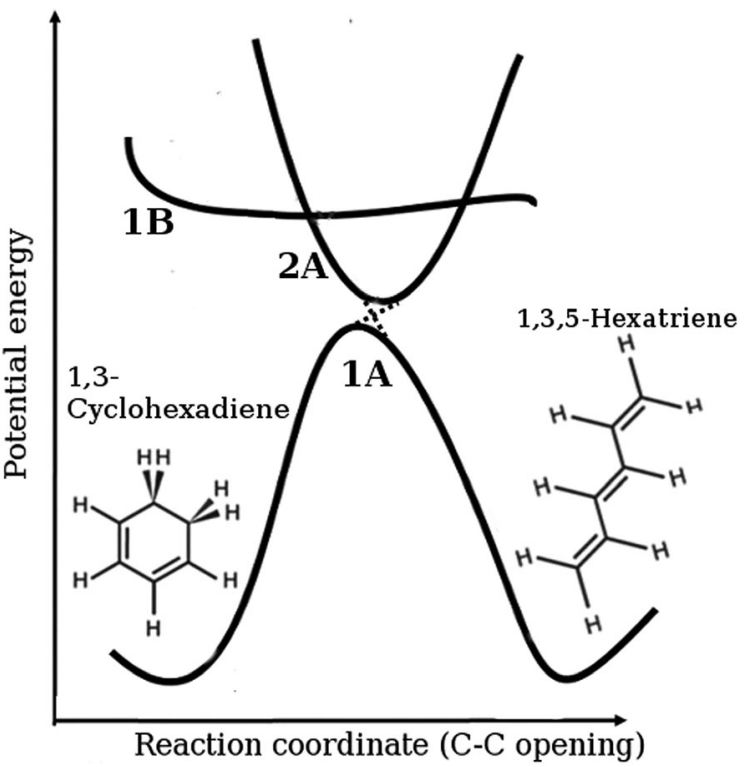

Fig. 1 Schematic representation of the ring-opening of 1,3-cyclohexadiene (CHD) to form 1,3,5-hexatriene (HT), including the diabatic electronic states involved in the process $(1 \mathrm{~A}$ is the ground state, $1 \mathrm{~B}$ the bright state, and $2 \mathrm{~A}$ the spectroscopically dark state). The molecule is vertically excited from the ring-closed potential well shown on the left-hand side of the ground state onto the $1 \mathrm{~B}$ state. It then progresses onto the $2 \mathrm{~A}$ state and then via the $2 \mathrm{~A} / 1 \mathrm{~A}$ conical intersection back onto the ground state in either the ring-open or the ring-closed form.

Interest in this reaction is driven by its role as a model system for modern mechanistic photochemistry. ${ }^{9-11}$ The CHD ringopening is a prototypical pericyclic reaction and has contributed to 
the development and corroboration of the Woodward-Hoffman rules, ${ }^{13}$ which predict the stereochemical outcome for pericyclic reactions by correlating the first excited state of the reactant with the first excited state of the product and the ground state of the reactant with a doubly excited state of the product, and vice versa. This yields a pericyclic minimum in the first excited state, which acts as photochemical funnel that provides an efficient path for internal conversion. ${ }^{14-16}$

The reaction has been studied extensively using a wide array of time-resolved techniques in solution and gas phase, including resonance Raman spectroscopy, ${ }^{17-19}$ transient absorption, ${ }^{12,20,21}$ photoelectron spectroscopy, ${ }^{2-24}$ and dissociative intense-field ionisation. ${ }^{25-27}$ Computational studies include electronic structure calculations ${ }^{16,28-33}$ and dynamics, ${ }^{34-42}$ as well as joint theoretical and experimental work..$^{23,43-45}$ It is notable that despite such extensive study, the understanding of the reaction is not complete. Apparent discrepancies include the branching ratio between ringopen and ring-closed products, possibly related to whether the experiment is performed in solution or gas phase. There is also an ongoing discussion regarding what dynamics occurs when the molecule is pumped at $200 \mathrm{~nm}$ rather than $267 \mathrm{~nm}^{46,47}$

Finally, and perhaps most importantly, CHD has been used extensively to demonstrate new techniques and concepts. This includes coherent control, both experiment ${ }^{43,48,49}$ and theory, ${ }^{7,50,51}$ and a range of new ultrafast imaging techniques ${ }^{52}$ such as electron diffraction, ${ }^{53-56}$ X-ray scattering, ${ }^{44,45}$ Coulomb explosion, ${ }^{57}$ and near-edge X-ray absorption fine structure (NEXAFS). ${ }^{58}$ The variety of complementary observations generated by these techniques holds great promise for tying down a complete understanding of this important and complex reaction, for instance by combining X-ray scattering and photoelectron data such as in ref. 59 .

In the present article, we explore the potential of tunable UV and XUV ultrafast lasers to probe photochemical dynamics in detail. Specifically, using a compact model of the dynamics excited by a $266 \mathrm{~nm}$ pump pulse, we examine the effect of changing the energy of the ionising probe pulse. The theoretical model is based on a reduced version of detailed quantum molecular dynamics simulations using the ab initio multiconfigurational Ehrenfest (AI-MCE) method, ${ }^{60}$ with a subset of representative trajectories extracted by comparison to X-ray scattering ${ }^{45}$ and photoelectron $^{59}$ experimental data. Time-dependent photoionisation cross-sections are calculated using the Dyson orbital approach. ${ }^{61}$ The resulting model allow us to predict the time-resolved photoelectron spectra for the ring-opening reaction and test the effectiveness of a variety of probe energies and the importance of including a sufficient number of ionisation channels in the theoretical description.

\section{Theory}

\subsection{Dynamics}

The nonadiabatic dynamics of photoexcited CHD molecules are simulated using the AI-MCE method. ${ }^{62,63}$ In this approach, the molecular wavefunction $|\Psi(t)\rangle$ is expanded as a sum of trajectory-guided Ehrenfest wavepackets, $\left|\psi_{k}(t)\right\rangle$, also referred to as trajectories,

$$
|\Psi(t)\rangle=\sum_{m=1}^{N_{\mathrm{trj}}} B_{m}(t)\left|\psi_{m}(t)\right\rangle,
$$

where $N_{\text {trj }}$ is the number of trajectories in the expansion and $B_{m}(t)$ are complex expansion coefficients. Collectively, these trajectories form an ansatz for solving the time-dependent Schrödinger equation. Each individual trajectory consists of electronic and nuclear components,

$$
\left|\psi_{m}(t)\right\rangle=\left[\sum_{i=1}^{N_{\mathrm{s}}} a_{m}^{i}(t)\left|\phi_{m}^{i}\right\rangle\right]\left|g_{m}(t)\right\rangle,
$$

where the nuclear Gaussian wavepacket $\left|g_{m}(t)\right\rangle$ is shared by the $N_{\mathrm{s}}$ electronic states, with each electronic wavefunction represented by $\left|\phi_{m}^{i}\right\rangle$. The population on each electronic state is given by $\left|a_{m}^{i}(t)\right|^{2}$. The nuclear wavepacket $\left|g_{m}\right\rangle=$ $\left|g_{1}^{m}\left(\mathbf{Q}_{1}^{m}(t), \mathbf{P}_{1}^{m}(t)\right)\right\rangle \ldots\left|g_{N_{\mathrm{at}}}^{m}\left(\mathbf{Q}_{N_{\mathrm{at}}}^{m}(t), \mathbf{P}_{N_{\mathrm{at}}}^{m}(t)\right)\right\rangle$ is a product of three-dimensional Gaussian coherent states for each atom where $N_{\text {at }}$ is the number of atoms. The labels $\mathbf{Q}_{\alpha}^{m}(t)$ and $\mathbf{P}_{\alpha}^{m}(t)$ are the coordinates and the momenta of a trajectory-guided three-dimensional Gaussian coherent state with width $\gamma_{\alpha}$. The component for the atom with index $\alpha$ in coordinate representation is then,

$$
\left\langle\mathbf{R}_{\alpha} \mid g_{\alpha}^{m}\right\rangle=\left(\frac{\gamma_{\alpha}}{\pi}\right)^{3 / 2} \mathrm{e}^{\left(-\frac{\gamma_{\alpha}}{2}\left(\mathbf{R}_{\alpha}-\mathbf{Q}_{\alpha}^{m}\right)^{2}+\frac{i}{\hbar} \mathbf{P}_{\alpha}^{m}\left(\mathbf{R}_{\alpha}-\mathbf{Q}_{\alpha}^{m}\right)+\frac{i}{2 \hbar} \mathbf{P}_{\alpha}^{m} \mathbf{Q}_{\alpha}^{m}\right)},
$$

where $\mathbf{R}_{\alpha}=\left(R_{\alpha x}, R_{\alpha y}, R_{\alpha z}\right)$ are the Cartesian nuclear coordinates in three dimensions.

Each individual trajectory, $\left|\psi_{m}(t)\right\rangle$, is propagated semiclassically along phase-space coordinates $\left\{\overline{\mathbf{Q}}_{\alpha}^{m}(t), \overline{\mathbf{P}}_{\alpha}^{m}(t)\right\}$ along with the Ehrenfest coefficients $a_{m}^{i}(t)$ and the expansion coefficients $B_{m}(t)$. The timedependent variation in the amplitudes $B_{m}(t)$ is due to coupling between trajectories. The propagation uses electronic potential energies and nonadiabatic couplings obtained from $a b$ initio electronic structure calculations. Readers interested in further details are directed to ref. 62 and 63. In this article, a reduced model based on a subset of Ehrenfest trajectories is used in the subsequent analysis of the photoelectron spectra (see Section 3.2 for details).

\subsection{Photoionisation}

The quantum molecular dynamics simulations are coupled to calculations of the photoionisation cross-sections and photoelectron spectra in the perturbative regime, corresponding to a Keldysh parameter $\gamma_{\mathrm{K}} \gg 1$, with $\gamma_{\mathrm{K}}=\omega \sqrt{2 I_{\mathrm{p}} / I}$, where $\omega$ is the angular frequency of the probe, $I_{\mathrm{p}}$ the ionisation potential, and $I$ the laser intensity. Assuming that lowest-order perturbation theory is valid, ${ }^{64,65}$ the $n$-photon ionisation rate is given by $\Gamma_{n}=\sigma_{n} I^{n}$, with $n$ the number of photons and $\sigma_{n}$ the generalized photoionisation cross section. Presently we consider single photon $n=1$ processes for which the photoelectron energy is,

$$
E_{k}=\hbar \omega-I_{\mathrm{p}}=\hbar^{2} k^{2} / 2 m_{\mathrm{e}},
$$


where $k$ is the asymptotic wavenumber and $m_{\mathrm{e}}$ the mass of the electron. This approximation does not account for intermediate resonances, such as those in resonance enhanced multiphoton ionisation processes for example. According to Fermi's golden rule ${ }^{66}$ the cross section under these conditions is,

$$
\sigma_{i}\left(E_{k}\right)=\frac{4 \pi^{2} e^{2} \omega}{c}\left|\hat{\varepsilon}\left\langle\Phi_{\mathrm{f}}\left(E_{k}\right)|\mathbf{r}| \phi_{i}\right\rangle\right|^{2},
$$

where $c$ is the speed of light, $e$ the charge of the electron, $\hat{\varepsilon}$ the unit polarisation vector of the probe field, and $\left\langle\Phi_{\mathrm{f}}\left(E_{k}\right)|\mathbf{r}| \phi_{i}\right\rangle$ the dipole transition moment with $\mathbf{r}$ the dipole moment operator, assuming the wavelength of the probe is sufficient for the dipole approximation, $\left|\phi_{i}\right\rangle$ the initial state, and $\left|\Phi_{\mathrm{f}}\left(E_{k}\right)\right\rangle$ the final energy-normalised continuum state which would normally satisfy incoming wave boundary conditions (see e.g. ref. 67-69). Any required integration over orientation quantum numbers is implicit.

We proceed by assuming that ionisation occurs between specific electronic states of the neutral molecule and the ion, and disregard coupling between ionisation channels or the vibrational states of the ion. Under these circumstances each contributing channel is identified by the initial electronic state $\phi_{i}$ and that of the molecular ion $\phi_{j}$. Upon ionisation, the electron is considered to be a free particle in the Coulomb field of the ion, and does not interact with the remaining core electrons. Following these approximations, one may use the Dyson orbital approach, ${ }^{61,70,71}$ which gives the dipole transition moment as,

$$
D_{i j}\left(E_{k}^{i j}\right)=\left\langle\Psi_{\mathrm{el}}^{k}|\mathbf{r}| \phi_{i j}^{\mathrm{D}}\right\rangle,
$$

where $\left|\Psi_{\mathrm{el}}^{k}\right\rangle$ the Coulomb wave function of the ejected electron and orientational averaging is implicit. ${ }^{61}$ The key quantity in eqn (6) is the Dyson orbital, $\left|\phi_{i j}^{\mathrm{D}}\right\rangle$, formed as the overlap of the initial $N$ electron wavefunction $\phi_{i}$ with the final $N-1$ electron wavefunction $\phi_{j}$. Integration over $N-1$ electron coordinates yields the one-electron Dyson orbital,

$$
\phi_{i j}^{\mathrm{D}}(\mathbf{r})=\sqrt{N} \int \phi_{i}\left(r_{1}, \ldots r_{N}\right) \phi_{j}\left(r_{2}, \ldots r_{N}\right) \mathrm{d} r_{2} \ldots \mathrm{d} r_{N},
$$

where strong orthogonality is assumed between the wavefunction of the departing electron and the remaining $N-1$ electrons. The dipole transition moment can be expressed in terms of the energy $\hbar \omega$ of the probe photon. The energy-resolved photoionisation cross section for ionisation from electronic state $i$ is the sum over all accessible states of the ion,

$$
\tilde{\sigma}_{i}\left(\hbar \omega, E_{k}\right)=\frac{4 \pi^{2} e^{2} \omega}{c} \sum_{j}\left|D_{i j}\left(\hbar \omega-I_{\mathrm{p}}^{i j}\right)\right|^{2} \delta\left(\hbar \omega-I_{\mathrm{p}}^{i j}-E_{k}\right),
$$

where $D_{i j}\left(E_{k}\right)=0$ for $E_{k}<0$ and $I_{\mathrm{p}}^{i j}=E_{j}-E_{i}$ is the ionisation potential for each channel as illustrated in Fig. 2. The expression given in eqn (8) corresponds to a stick-spectrum as a function of photoelectron energy $E_{k}$ with the relationship between $\hbar \omega$ and $E_{k}$ given by eqn (4). When calculating the total photoionisation cross section from the simulations, one must account for the

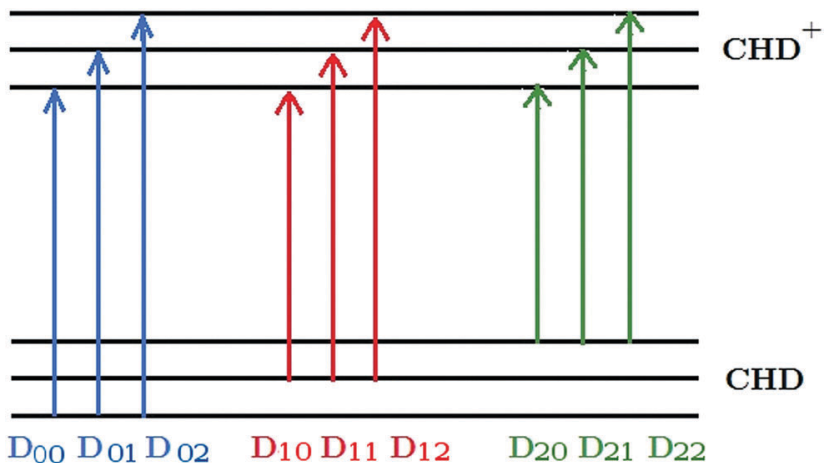

Fig. 2 Schematic depiction of the ionisation channels, $D_{i j}$, with ithe electronic state of the neutral CHD molecule $\left(S_{0}, S_{1}, S_{2}, i=0,1,2\right)$ and $j$ of the $\mathrm{CHD}^{+}$ion $\left(D_{0}, D_{1}, D_{2}, j=0,1,2\right)$. Each combination of $i$ and $j$ corresponds to a different Dyson orbital as shown in Fig. 4. Channels corresponding to ionisation from the ground state $S_{0}$ are shown in blue, from the first excited state $S_{1}$ in red, and from the second excited state $S_{2}$ in green. The ionisation potentials for each of the channels are $l_{p}^{i j}=E_{j}-E_{i}$, with $i$ and $j$ as above.

contribution of each quantum trajectory according to eqn (1) and (2),

$$
\tilde{\sigma}_{\mathrm{tot}}\left(\hbar \omega, E_{k}, t\right)=\sum_{m}^{N_{\mathrm{trj}}}\left|B_{m}(t)\right|^{2} \sum_{i}^{N_{\mathrm{s}}}\left|a_{m}^{i}(t)\right|^{2} \tilde{\sigma}_{i}^{m}\left(\hbar \omega, E_{k}, t\right) .
$$

Note that all factors, including the ionisation potential for each channel, are time-dependent. The photoelectron spectrum corresponds to the probability of detecting an electron with a certain kinetic energy. We convolute the signal to account for the energy distribution of the probe pulse. Expressing the photoionisation cross section in terms of the photoelectron kinetic energy, the photoelectron signal then becomes,

$$
\sigma_{\mathrm{tot}}\left(E_{k}, t\right)=\sum_{m}^{N_{\mathrm{trj}}}\left|B_{m}(t)\right|^{2} \sum_{i}^{N_{\mathrm{s}}}\left|a_{m}^{i}(t)\right|^{2} \int \tilde{\sigma}_{i}^{m}\left(\hbar \omega, E_{k}, t\right)|\varepsilon(\hbar \omega)|^{2} \hbar \mathrm{d} \omega,
$$

where $|\varepsilon(\hbar \omega)|^{2}$ is the intensity profile of the probe pulse.

\section{Computational details}

\subsection{Dynamics}

The AI-MCE ${ }^{72}$ dynamics are calculated using the adiabatic electronic energies, gradients, and nonadiabatic couplings calculated on-the-fly at the SA3-CAS(6,4)-SCF/cc-pVDZ level of theory using the ab initio electronic structure package MOLPRO. ${ }^{73}$ The choice of the SA3-CAS $(6,4)$ active space is guided by previous investigations ${ }^{74}$ that demonstrate that it provides reasonable energy and gradient information on the relevant electronic states for the dynamics. The simulations assume that the vertical excitation is instantaneous, with the initial phase-space coordinates on the excited state generated using the vibrational Wigner distribution ${ }^{75}$ for the $\nu=0$ vibrational state on the electronic ground state,

$$
W_{0}^{\mathrm{HO}}=\frac{1}{\pi \hbar} \prod_{i} \exp \left(-\frac{m_{i} \omega_{i}}{\hbar}\left(Q_{i}-Q_{i}^{0}\right)^{2}-\frac{P_{i}^{2}}{m_{i} \hbar \omega_{i}}\right),
$$


where $\omega_{i}$ is the frequency of each normal mode and $m_{i}$ is the reduced mass. The phase-space coordinates are generated from the total distribution using Monte-Carlo sampling. A set of 100 trajectories are propagated for 200 fs with time-step 0.1 fs. A note regarding the diabatic/adiabatic notation for the electronic states is in order. The trajectories are propagated on adiabatic electronic states denoted $S_{0}, S_{1}$, and $S_{2}$. Accordingly, the wavepacket is initially pumped onto the $S_{1}$ state, which in the Franck-Condon region corresponds to the diabatic $1 \mathrm{~B}$ state. As the wavepacket evolves, it reaches a $2 \mathrm{~A} / 1 \mathrm{~B}\left(\mathrm{~S}_{2} / \mathrm{S}_{1}\right)$ conical intersection, at which point part of the wavepacket may populate the $S_{2}$ state. The main portion of the wavepacket remains on the $S_{1}$ state, continuing to a section of the $S_{1}$ state with $2 \mathrm{~A}$ character, and then decays back to the ground state via the $2 \mathrm{~A} / 1 \mathrm{~A}\left(\mathrm{~S}_{1} / \mathrm{S}_{0}\right)$ conical intersection.

\subsection{Compact model}

The calculations of photoionisation cross sections along each of the Ehrenfest trajectories is computationally expensive, and importantly, the purpose of our analysis is to evaluate the link between dynamics, reaction paths, and the energy of the probe pulse on the signals, rather than the best-possible accuracy of the predicted total signal. We therefore define a compact model of the dynamics based on the full set of trajectories, but which consists of a small subset of these. The subset is determined by minimising the discrepancy between the signal predicted by the full set of trajectories and experimental data from ultrafast X-ray scattering, with the (in this case time-independent) expansion coefficients $B_{m}$ used as variables in the optimisation as described in ref. 45. It is found that only four trajectories are sufficient to account for $>70 \%$ of the scattering signal. The model obtained was independently validated against resonant photoionisation time-dependent experimental data, again finding good agreement. ${ }^{59}$ Further insight into the effectiveness of the model is obtained from a cluster analysis of the full set of trajectories, which shows that the actual dynamics can be represented by a much smaller set of trajectories corresponding to a small number of self-similar sets. ${ }^{76}$ In effect, the four trajectories in the model represent effective reaction coordinates. The main benefit of this approach is that it dramatically reduces the cost of otherwise expensive calculations and enables a detailed analysis of the correlation between the features of each trajectory and the resulting time-resolved photoelectron spectra. The four trajectories used hereon are labeled a, b, c and d. They are described in detail in Section 4.2, but broadly, the two trajectories labeled as a and c correspond to ring-opening dynamics, while the two trajectories labeled as b and d correspond to ring-closed dynamics.

\subsection{Photoionisation}

For the calculations of the ionisation cross section and the photoelectron spectra we take into account the ground and two excited states of the neutral molecule and the target ion, providing nine independent ionisation channels. These channels are shown in Fig. 2 and are labeled as $D_{i j}$, where $i$ and $j$ identify the initial and final states. In all figures hereon, we show the channels associated with states $S_{0}, S_{1}$ and $S_{2}$, in blue, red and green, respectively. Note that all photoionisation cross sections are evaluated at the center of each Gaussian wavepacket (see discussion of BAT approximation e.g. in ref. 60).

The Dyson orbitals are calculated using Q-Chem, ${ }^{77}$ with initial and final state electronic wavefunctions calculated at the EOM-CC/6-311G* level of theory. At each point, the output files generated with $\mathrm{Q}$-Chem are used to extract the ionisation energies. The cross sections for each channel are then calculated using the ezDyson code. ${ }^{70}$ The free electron wavefunction is expanded in a series of partial waves with angular momentum $l=0-5$ using a spatial grid with 251 grid points extending to a radius of 25 Bohr. Cross sections are calculated at 41 values of the kinetic energy in the range $0.3-15 \mathrm{eV}$. All calculations are performed in the laboratory frame assuming rotational averaging. The computations of the photoionisation cross sections are automated using a script, which reads the molecular geometry and electronic state populations from the simulations, calculates the Dyson orbitals using Q-Chem, and then runs ezDyson to obtain the cross sections. The ionisation cross sections are calculated every 10 fs for the dynamics. The signal is convoluted by the intensity profile of the probe laser $|\varepsilon(\hbar \omega)|^{2}$, which is assumed to be Gaussian with FWHM $0.3 \mathrm{eV}$ and centered at the probe energy $\hbar \omega_{0}$.

\section{Results}

\subsection{Photoionisation validation}

To validate the calculated photoionisation cross sections, we compare the calculations for the neutral ground-state CHD molecule at the equilibrium $(t<0)$ geometry with experimental data from photoionisation mass spectrometry. ${ }^{78}$ In the experiments, the photon-energy dependent yield of the $\mathrm{C}_{6} \mathrm{H}_{8}{ }^{+}$(so-called channel 1) and $\mathrm{C}_{6} \mathrm{H}_{7}{ }^{+}$(channel 2) ionisation products are measured, which are taken to be proportional to the ionisation cross sections. The experimental yields are compared to energy-integrated calculated cross sections for the channels $D_{00}, D_{01}$, and $D_{02}$ (i.e. the channels that correspond to ionisation of the neutral ground state molecule in Fig. 2). The lowest ionisation potential is $8.3 \mathrm{eV}$, corresponding to the channel $D_{00}$. After the initial increase in cross section around the first ionisation potential, both experiment and computations show a plateau up to $11 \mathrm{eV}$. At $11 \mathrm{eV}$, the $D_{01}$ and $D_{02}$ channels become open and a large increase in ionisation cross section is observed. The two channels $D_{01}$ and $D_{02}$ have similar ionisation potentials and their cross sections are also similar as can be seen in Fig. 3a. In the experimental data, the signal in the so-called channel 2 corresponding to detection of $\mathrm{C}_{6} \mathrm{H}_{7}{ }^{+}$, also appears at approximately $11 \mathrm{eV}$, which strongly suggests that the experimental channel 2 correlates with the ionisation channels $D_{01}$ and $D_{02}$. This indicates that ionisation into excited electronic states of the $\mathrm{CHD}^{+}$cation eventually leads to dissociative decay and fragmentation of the cation.

A second validation undertaken is to compare the calculated photoelectron spectrum of the ground state CHD to previous experimental spectra. ${ }^{22,79}$ Fig. 3 b, where the calculated data has 


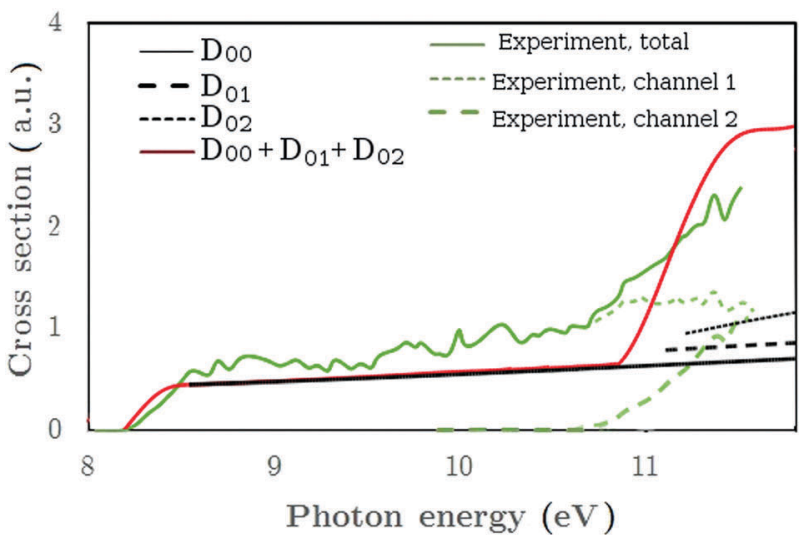

(a) Total ionisation cross section

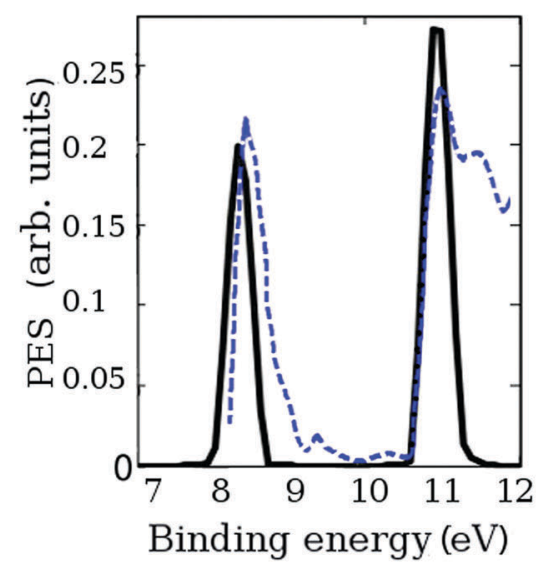

(b) Photoelectron spectrum

Fig. 3 Validation of calculated photoionisation cross sections by comparison to experimental data. (a) Total interpolated photoionisation cross section from the ground state (shown in red) compared to experimental data from Wang et al. ${ }^{78}$ (in green). The black lines show the calculated partial cross sections. (b) Calculated photoelectron spectrum (in black) compared to experimental data (blue dashed line) from Adachi et al. ${ }^{22}$ been scaled by a single number for comparison, shows good agreement between experiment and theory both in terms of the positions of the two peaks and their relative intensity. The difference in the broadness of the peak at higher energy is due to the presence of higher excited states of the ion that are not included in the calculation. In summary, the two comparisons used to validate the Dyson-orbital calculations indicate that the approach provides a reasonably accurate description of the photoionisation cross sections over the energy range considered, at least for the ground state of CHD.

Fig. 4 shows the calculated Dyson orbitals as the molecule progresses along trajectory a. The top row shows the Dyson orbitals corresponding to each of the ionisation channels in the ground state geometry $(t<0)$, while the bottom row shows the same Dyson orbitals at time $t=180 \mathrm{fs}$ in the ring-opening trajectory a. The shape of the orbitals clearly indicates that there might be significant anisotropy in the photoionisation signal, however, this is currently not accounted for as the signal is integrated over all orientations.

\subsection{Dynamics}

In the following, we discuss the four trajectories used to represent the overall dynamics of the photoexcited CHD. As discussed in Section 3.1, these should be seen to represent effective reaction coordinates. The trajectories are labelled a-d. Trajectories (a, c) relate to the main ring-opening pathways, while trajectories $(\mathrm{b}, \mathrm{d})$ to ring-closing pathways. The changes in molecular geometry for each trajectory can be characterized by the $\mathrm{C}_{1}-\mathrm{C}_{6}$ bond distance, corresponding to the bond that is broken during ring-opening, and shown in red in Fig. 5 for each of the trajectories. The black curves in the same figure show how three of the other $\mathrm{C}-\mathrm{C}$ bonds change with time (these curves have been shifted by $+3 \AA$ for clarity). The ring-closed trajectories (b, d) exhibit strong oscillatory behavior in the $\mathrm{C}_{1}-\mathrm{C}_{6}$ distance that decreases over time as the energy disperses to other internal degrees of freedom. The ring-opening trajectories (a, c) demonstrate rapid lengthening of the $\mathrm{C}_{1}-\mathrm{C}_{6}$ bond with little

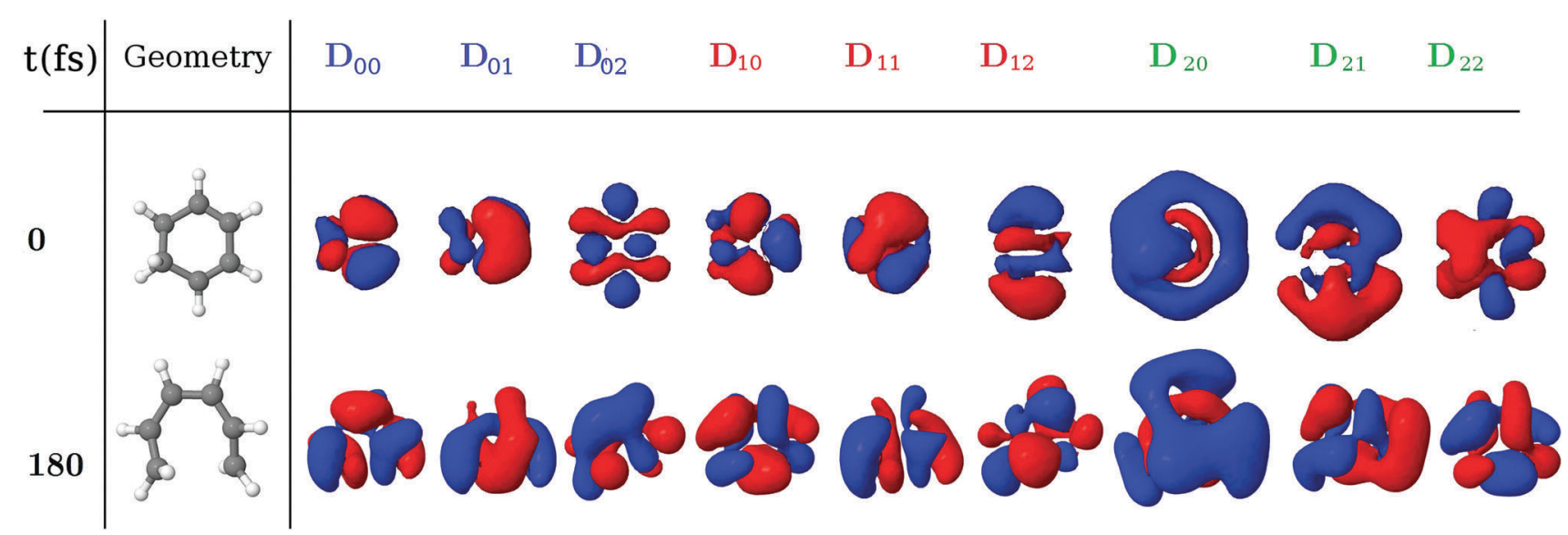

Fig. 4 Dyson orbitals for each of the ionisation channels $D_{i j}$, where $i$ is the initial neutral electronic state and $j$ is the electronic state of the ion. The orbitals are shown at equilibrium geometry ( $t=0 \mathrm{fs}$, top row), and at time $t=180 \mathrm{fs}$ in the ring-opening trajectory a (bottom row). The first column shows the molecular geometry in each case. 

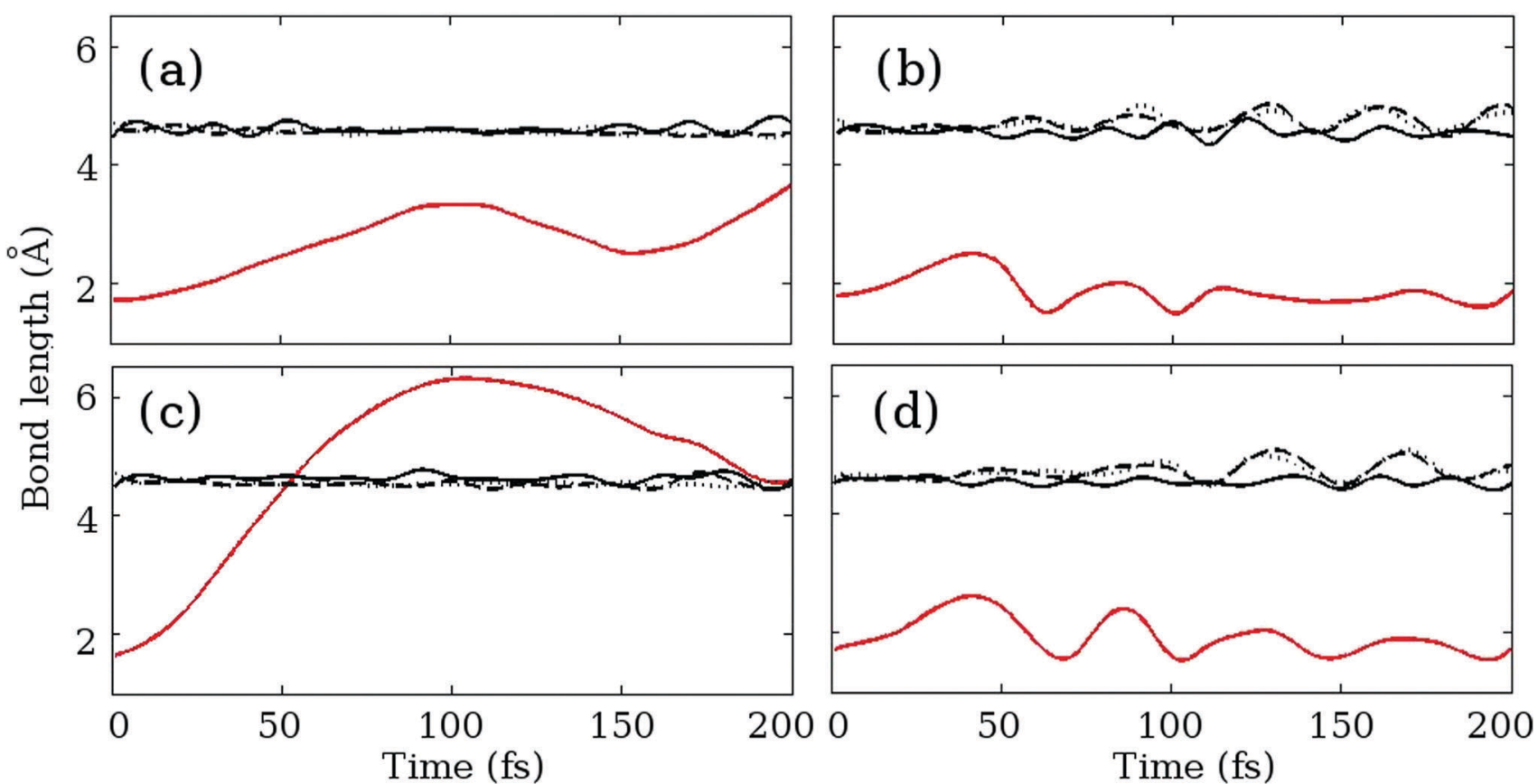

Fig. 5 Distances between the carbon atoms as a function of time for each of the trajectories ( $a, b, c$ and d) shown in the corresponding panels a-d. The distance between carbon atoms $C_{1}$ and $C_{6}$, corresponding to the bond that is broken in the ring-opening reaction, is shown in red. Note that trajectories ( $a$ and $c$ ) are ring-opening and ( $b$ and $d$ ) are ring-closed. Three further nearest-neighbour $\mathrm{C}_{i}-\mathrm{C}_{i \pm 1}$ distances are shown in black, but have been shifted by $+3 \AA$ to avoid congestion in the plots. The panels are labeled as follows: (a) trajectory a (direct to open; weight 0.2), (b) trajectory b (closed; weight 0.19), (c) trajectory c (rapid open; weight 0.18 ), and (d) trajectory d (closed; weight 0.14 ).

variation in the other $\mathrm{C}-\mathrm{C}$ bonds. The rapid $\mathrm{C}_{1}-\mathrm{C}_{6}$ extension in trajectory c quickly leads to large $\mathrm{C}_{1}-\mathrm{C}_{6}$ distances of $\approx 6 \AA$. The slower ring-opening in trajectory a, while still leading to bond breaking, maintains much closer proximity of $\mathrm{C}_{1}$ and $\mathrm{C}_{6}$ over the course of the simulation.

The time-dependent electronic state populations plotted in Fig. 6 provide an explanation for some of these differences. In trajectory a the majority of the dynamics occurs on the $S_{1}$ surface. There is a nonadiabatic transient transfer of population to the $S_{2}$ and $S_{0}$ states between $20 \mathrm{fs}$ and $60 \mathrm{fs}$, but a large fraction of the population remains on the $S_{1}$ surface while the $\mathrm{C}_{1}-\mathrm{C}_{6}$ bond breaks, such that this trajectory can be considered an excited-state ring-opening. At a comparatively late stage in the dynamics ( $\approx 150 \mathrm{fs}$ ) approximately half of the $\mathrm{S}_{1}$ population decays to the ground state. As for the other three trajectories (b, c and d), these follow a more typical pattern with a rapid transfer of population into the ground $S_{0}$ state. In each case the ground state is reached within $50 \mathrm{fs}$ following excitation. All four trajectories demonstrate limited transfer of population to the $\mathrm{S}_{2}$ state at early times, occurring when the wavepacket propagating on the $S_{1}$ state reaches the region of the $S_{2} / S_{1}$ conical intersection. In this region, the $S_{1}$ state changes from predominantly $1 \mathrm{~B}$ to predominantly $2 \mathrm{~A}$ character. For trajectory $\mathrm{c}$, which leads to ring-opening, exchange of population between the electronic states occurs along the entire pathway, while for trajectories $\mathrm{b}$ and $\mathrm{d}$ the main transfer occurs at around $t=150 \mathrm{fs}$. In particular for trajectories (b, c and d) the rapid electronic state relaxation leads to excess vibrational energy that in trajectory $\mathrm{c}$ is transferred into motion of molecule that drives $\mathrm{C}_{1}$ and $\mathrm{C}_{6}$ rapidly away from each other. For the trajectories that remain closed the excess energy disperses into multiple vibrations around the ring leading to rapid and large amplitude changes in the $\mathrm{C}-\mathrm{C}$ distances. The weighted average of the populations from all four trajectories combined, shown as a function of time in Fig. 7, agrees with the accepted view of the CHD ringopening reaction described in Section 1. Comparison of Fig. 6 and 7 also highlights that the total populations mask the underlying mechanisms at play and that examination of the individual trajectories contributes to a more detailed picture of the dynamics.

\subsection{Time-resolved photoelectron spectra}

In order to analyse photoionisation signals it is important to comprehend the changes in ionisation potential over the course of the reaction. The changes in ionisation potential control the measured photoelectron energy for any given probe-photon energy, and are critical for the assignment of features within the spectrum. The calculated ionisation potentials for all nine ionisation channels considered here are shown in Fig. 8 for each of the four trajectories. Generally, the ionisation potentials associated with the excited states, $S_{1}$ and $S_{2}$, show more variation than those associated with $\mathrm{S}_{0}$. At early times the rapid changes in the $\mathrm{C}_{1}-\mathrm{C}_{6}$ distance lead to pronounced shifts in ionisation potential. For trajectories (b and d) changes in the ionisation potential at longer times, $t>100 \mathrm{fs}$, correlate with the rapid oscillations in the remaining $\mathrm{C}-\mathrm{C}$ bonds as seen in Fig. 5 . 

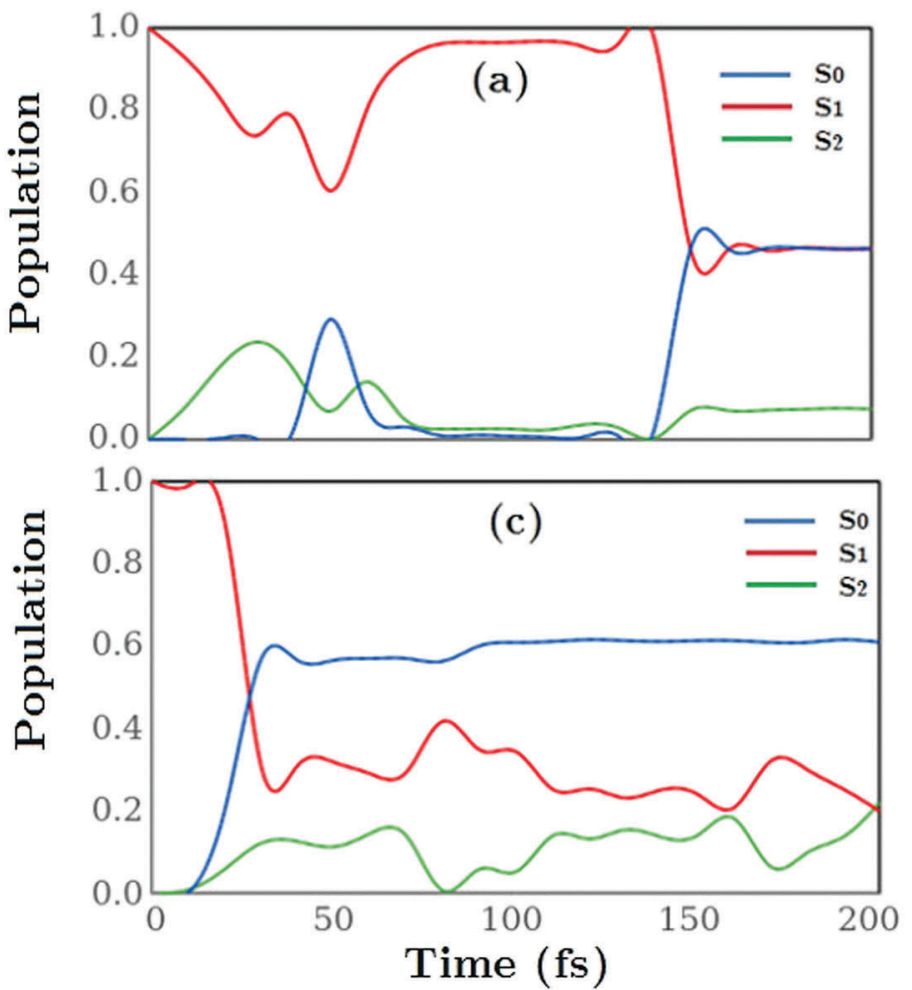
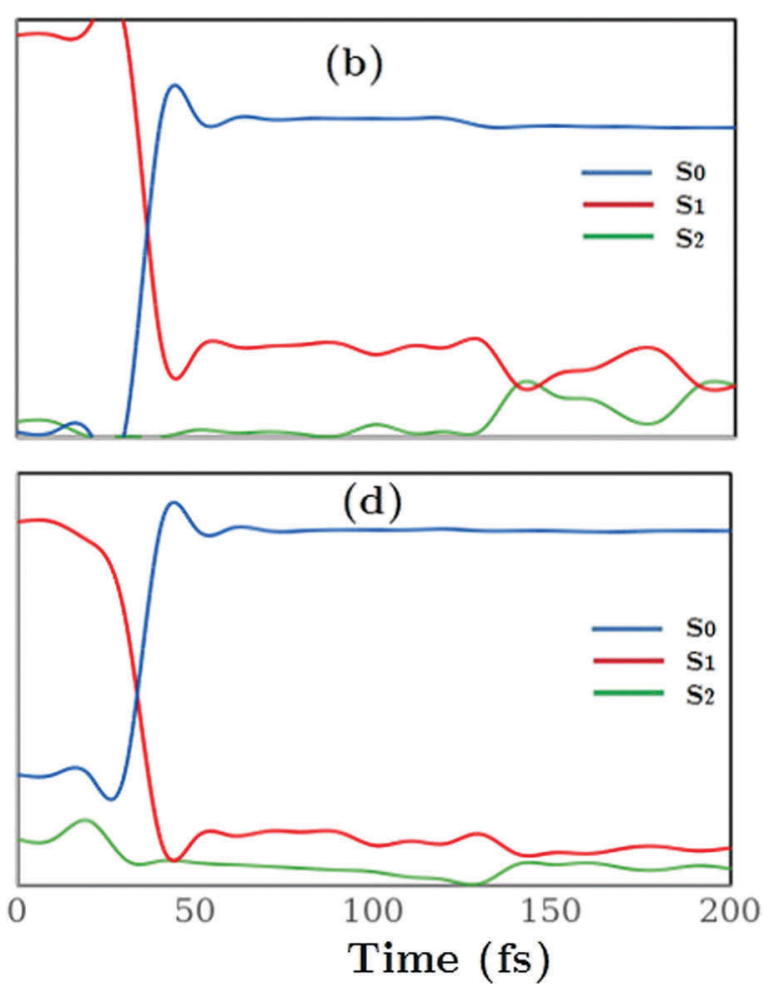

Fig. 6 Populations $\left|a_{k}^{i}(t)\right|^{2}$ in the electronic states $i=S_{0}, S_{1}$, and $S_{2}$ as a function of time for the four trajectories (a, b, c and d) shown in panels a-d (labels correspond to Fig. 5).

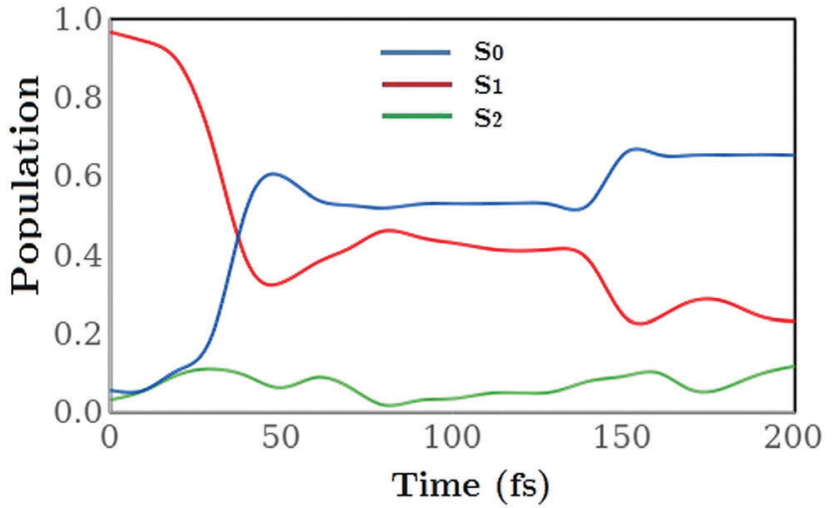

Fig. 7 The effective electronic state population as a function of time for all four trajectories combined. The population of the $S_{2}$ state remains low throughout.

We proceed to examine the time-dependent photoionisation cross sections as a function of photon energy. The total cross sections are calculated by integrating expression eqn (9) over $E_{k}$, and are shown as a function of the photon energy $\hbar \omega$ and the time $t$ in Fig. 9. The ionisation cross section for probe energies below $8 \mathrm{eV}$ decreases once the molecule relaxes to the ground state via the conical intersection at around $t=30-50 \mathrm{fs}$. This highlights one of the key drawbacks associated with UV photoelectron spectroscopy, in that the lifetimes measured often relate to the time the wavepacket remains in an ionisable region of the excited state potential and not necessarily the fundamental lifetimes of the molecule. In contrast, the increase of population on the ground state for $t>50 \mathrm{fs}$ leads to a large effective cross sections for high energy $(>10 \mathrm{eV})$ probe photons.

The time-dependent photoelectron spectrum at probe energies $2 \mathrm{eV}, 6 \mathrm{eV}, 9.2 \mathrm{eV}$ and $15 \mathrm{eV}$ as calculated by eqn (10) is shown in Fig. 10. The $2 \mathrm{eV}$ probe is chosen to demonstrate the utility of a highly selective probe, the $6 \mathrm{eV}$ probe represents to the maximum energy attainable from conventional non-linear optics, while $9.2 \mathrm{eV}$ and $15 \mathrm{eV}$ probes are within the range covered by femtosecond filamentation sources, high-harmonic generation, or seeded freeelectron laser sources ${ }^{80-83}$ and approach energies sufficiently high to be considered universal photoionisation probes.

The spectra shown in Fig. 10 show increasing complexity and structure, the greater the probe energy. The signal from the $2 \mathrm{eV}$ probe shown in Fig. 10a is rather weak, nearly two orders of magnitude lower in intensity and less complex than the higher energy probes, but is selective in its sensitivity to the transient population in the $S_{2}$ state. The $6 \mathrm{eV}$ probe in Fig. 10b provides a measure of the dynamics in the $S_{1}$ and $S_{2}$ excited states, in turn. The spectrum shifts to higher binding energies during the first 50 fs as the $\mathrm{C}_{1}-\mathrm{C}_{6}$ bond extends and the wavepacket moves down towards the conical intersection that funnels the wavepacket onto the ground state. Once the wavepacket is on the $\mathrm{S}_{0}$ state, the $6 \mathrm{eV}$ probe can no longer ionise the wavepacket and the signal decreases in intensity. The $6 \mathrm{eV}$ probe thus provides no information on the dynamics in the ground state or the formation of the final reaction products. 

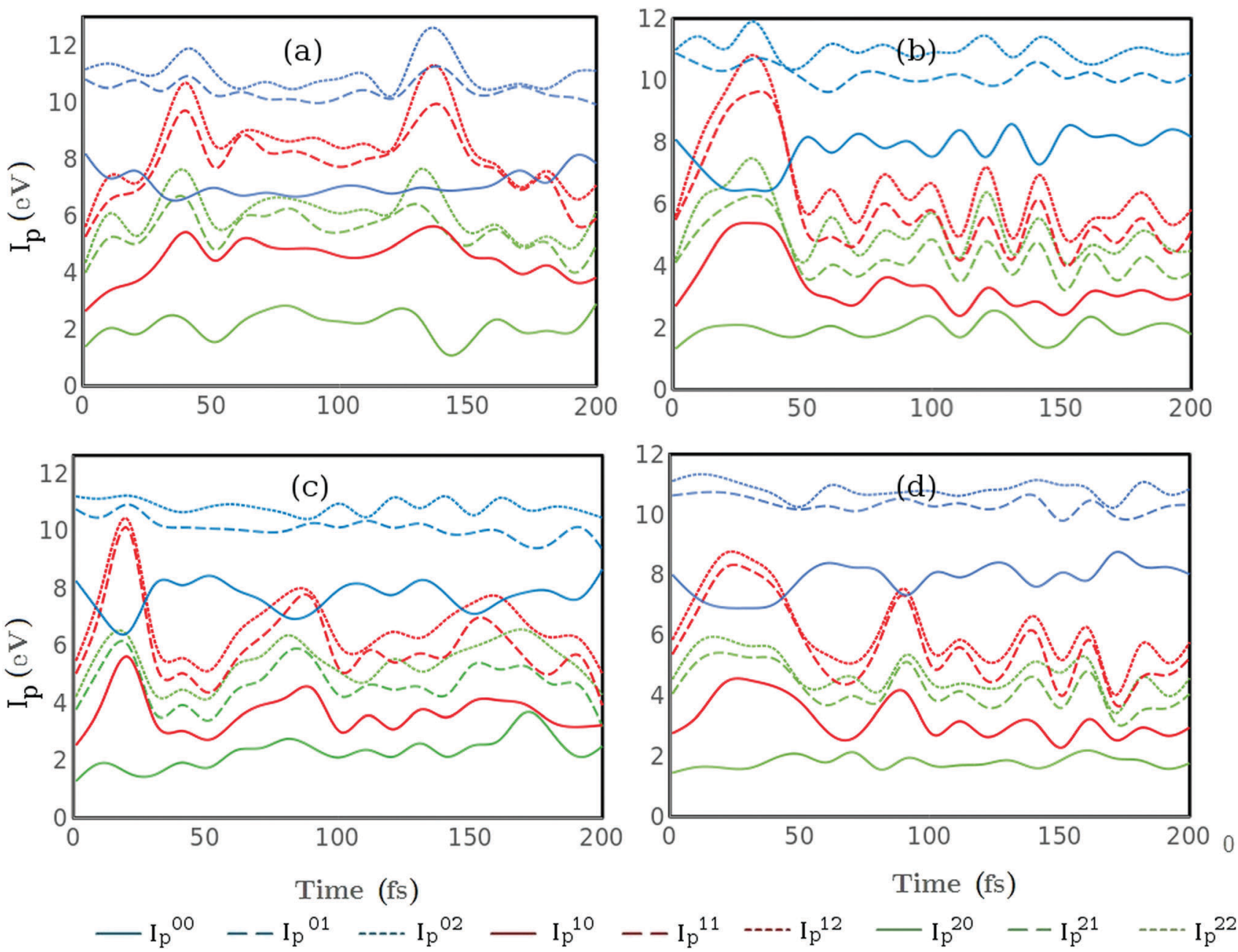

$\mathrm{Ip}^{10}$

$-I_{p}^{11}$

$\mathrm{Ip}^{12}$

$-\mathrm{Ip}_{\mathrm{p}}{ }^{20}--\mathrm{Ip}^{21}$

Fig. 8 Ionisation potentials $I_{p}^{i j}=E_{j}-E_{i}$ as a function of time for each of the trajectories (a, b, c and d) shown in the corresponding panels a-d (labels as in Fig. 5). $E_{i}$ is the potential energy of $C H D$ in states $i=0,1,2\left(S_{0}, S_{1}\right.$ and $\left.S_{2}\right)$ and $E_{j}$ that of $C H D^{+}$in states $j=0,1,2\left(D_{0}, D_{1}\right.$ and $\left.D_{2}\right)$, resulting in nine channels overall. The channels that originate in $S_{0}, S_{1}$ and $S_{2}$, are shown in blue, red and green, respectively, and channels that correspond to excited states of $\mathrm{CHD}^{+}(j>0)$ are shown as dashed lines.

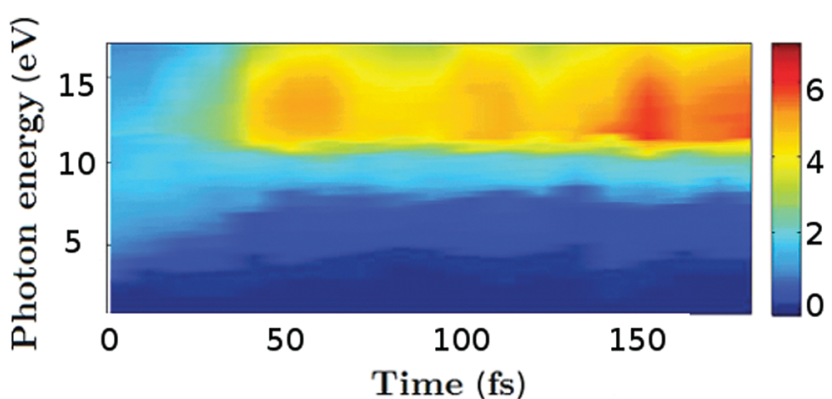

Fig. 9 Total photoionisation cross section in atomic units for all four trajectories as a function of time (fs) and probe photon energy (eV).

Increasing the probe energy beyond $6 \mathrm{eV}$ provides a more universal measure of the dynamics. The $9.2 \mathrm{eV}$ and $15 \mathrm{eV}$ probes, shown in Fig. 10c and d, give rise to more complex spectra which include contributions from the $\mathrm{S}_{0}$ ground state. The redistribution of population at the conical intersection is seen in Fig. 10c and d at around $40 \mathrm{fs}$ as a splitting of the main photoelectron feature, with the remaining $\mathrm{S}_{1}$ population contributing to a feature at around $5 \mathrm{eV}$, while the signal related to the ground state continues to shift to higher binding energies. Notably, ionisation from $\mathrm{S}_{0}$ contributes the most to the signal. Importantly, the highest energy $15 \mathrm{eV}$ probe is capable of distinguishing the CHD and HT forms on the $S_{0}$ potential energy surface. ${ }^{79,81}$ The CHD and HT photoionisation spectra are almost identical for the HOMO peak, but starting with HOMO-1 the spectra are clearly distinguishable. ${ }^{81}$

Fig. 11 presents the decomposition of the time-resolved photoelectron signal obtained with the $15 \mathrm{eV}$ probe in Fig. 10d in terms of the contributions from each of the four trajectories $\mathrm{a}-\mathrm{d}$ (shown in corresponding panels $\mathrm{a}-\mathrm{d}$ ). The partial spectra for the two ring-closed trajectories (b and d) are more intense for $t>50 \mathrm{fs}$, which relates to the more complete transfer of population to the electronic ground state $S_{0}$ for the ring-closed trajectories on the time-scale of the simulations and that the cross section for photoionisation is large in the $S_{0}$ state. 


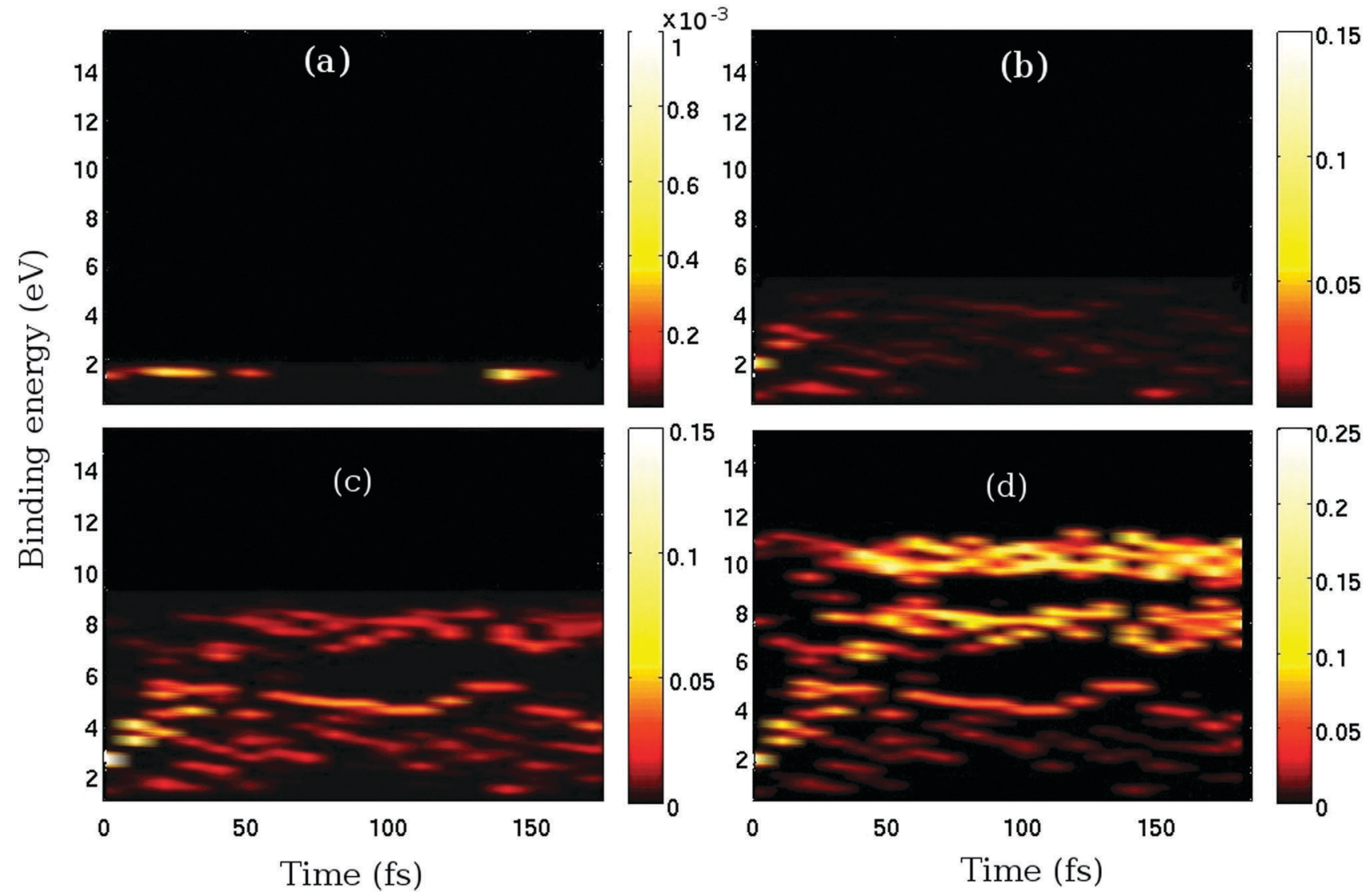

Fig. 10 Total photoelectron spectra (for all four trajectories combined) at probe energy: (a) $2 \mathrm{eV}$, (b) $6 \mathrm{eV}$, (c) $9.2 \mathrm{eV}$, and (d) $15 \mathrm{eV}$. The probe pulse envelope has Gaussian shape with bandwidth $\approx 0.3 \mathrm{eV}$.

The overall slower decay of the excited state population in the ring-opening (a, c) trajectories also implies that spectroscopic assessment of the branching ratio between CHD and HT should be done at asymptotically long times rather than at short times. All four trajectories exhibit a rapid increase in the photoelectron binding energy $E_{\mathrm{b}}$ at early times, corresponding to the wavepacket sliding down the potential-energy funnel towards the conical intersection and the subsequent internal conversion at the conical intersection. The intense higher-energy bands for the ring-closed trajectories (b, d) exhibit distinct beats. Out of the two ring-opening trajectories $(a, c)$, the electronic state population dynamics for trajectory $\mathrm{c}$ is more similar to the ring-closed trajectories (b, d), and correspondingly it also exhibits features at low- and mid-energy that are qualitatively similar to those of the spectra for the ring-closed trajectories. Finally, the distinct character of all four time-resolved spectra emphasises the challenge in deconvoluting and interpreting the total spectrum shown in Fig. 10d previously.

To quantify the importance of including ionisation channels corresponding to excited electronic states of the cation when predicting photoelectron spectra obtained with high-energy probes, we evaluate separately the contribution to the total signal from ionisation channels corresponding to the ground and excited states of the cation, as shown in Fig. 12, at 9.2 and
$15 \mathrm{eV}$ probe energies. At $9.2 \mathrm{eV}$ the fraction of the signal due to excited cation states remains comparatively small, with a rather stable $30 \%$ fraction at all times. For the $15 \mathrm{eV}$ probe, the excited states of the cation become important, and constitute $>50 \%$ of the signal at times $t>50 \mathrm{fs}$. Although the importance of the excited-state channels is indisputable for high-energy probes, it is interesting to note that at $t<50 \mathrm{fs}$, the excited cation fraction for the $15 \mathrm{eV}$ probe is as low as $15 \%$, which is a consequence of the ionisation potentials and photoionisation cross sections for molecular geometries close to the ring-closed equilibrium geometry.

Finally, it is worth pointing out that the most direct comparison to experiment lies with the work of Adachi et al. ${ }^{22}$ who used a HHG probe of $13.6 \mathrm{eV}$. Those measurements show an early shift in the peak position associated with the excited state population which is taken as the transfer of population from the $S_{1}$ to the $S_{2}$ electronically excited state. The energy of the excited state bands at a binding energy around $5 \mathrm{eV}$ which matches our calculated values associated the excited state driven dynamics on trajectory a. The measurements show a delayed increase in the ground state population of the ground state CHD and HT, although the measurements are hindered by an experimental artifact as discussed in ref. 22 that obscures the early-time dynamics at $<200$ fs, precluding direct comparison. 

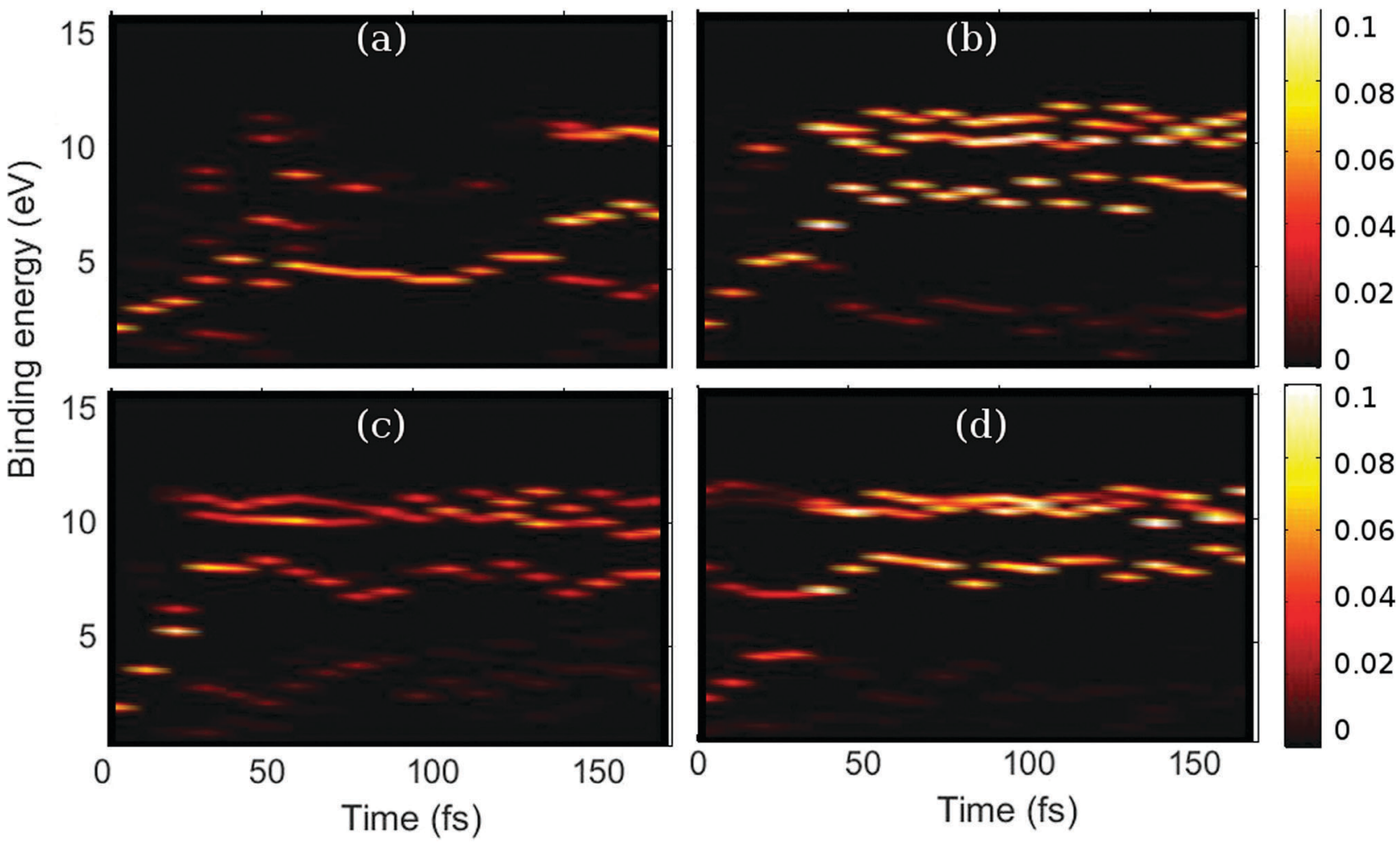

Fig. 11 Photoelectron spectra for each of the trajectories ( $a, b, c$ and d) shown in corresponding panels a-d. The signal intensity is shown as a function of time and photoelectron binding energy $E_{\mathrm{b}}=\hbar \omega_{0}-E_{k}$. The spectra in all four panels are calculated with the $15 \mathrm{eV}$ probe (the corresponding total signal is shown in Fig. 10d). Panels ( $a$ and c) correspond to ring-opening, and panels (b and d) to ring-closed trajectories as before, matching the labels in Fig. 5.

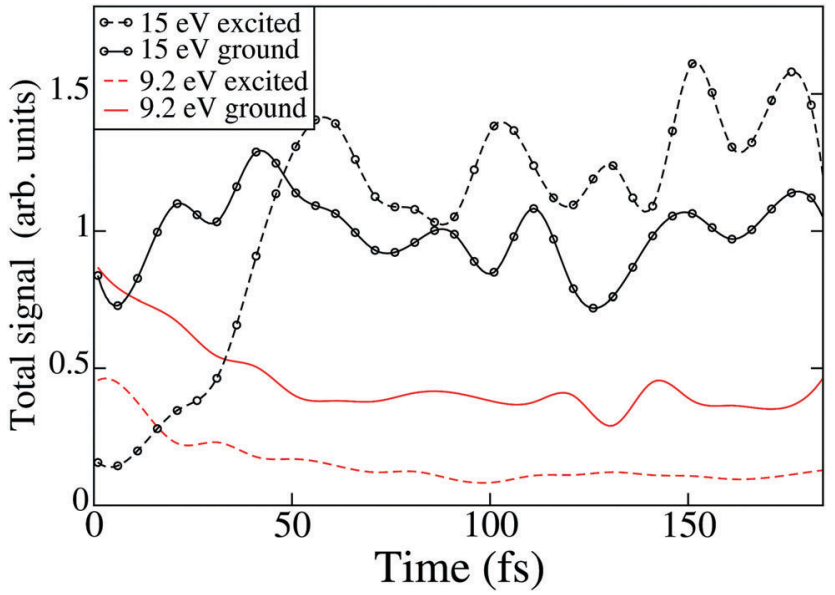

Fig. 12 Total photoelectron signals (calculated for all four trajectories combined) as a function of time for the $9.2 \mathrm{eV}$ and $15 \mathrm{eV}$ probes. The total signal is split into the contribution from ionisation channels corresponding to the ground state of the cation (channels $D_{00}, D_{10}$, and $D_{20}$ ) and the contribution from the excited states of the cation $\left(D_{i j}\right.$ with $j>0$ and any $\left.i\right)$. The contribution from the excited states of the cation is approximately $30 \%$ for the $9.2 \mathrm{eV}$ probe at all times, and $>50 \%$ for $t>50$ fs for the $15 \mathrm{eV}$ probe.

\section{Conclusions}

Using a compact model based on AI-MCE quantum molecular dynamics $^{45}$ and a Dyson orbital approach for calculating ionisation cross sections, ${ }^{61}$ we have simulated time-resolved photoelectron spectra for the ring-opening reaction that transforms 1,3cyclohexadiene (CHD) to hexatriene (HT). The calculations of photoelectron cross sections are validated against experimental CHD spectra, which provides an opportunity to assign the so-called channel two observed by mass spectrometry ${ }^{78}$ to excited-state fragmentation of the $\mathrm{CHD}^{+}$cation. In the time-resolved spectra, the energy shift in the photoelectron spectrum at early times provides a direct reflection of the photochemical funnel which guides the initially excited wavepacket towards the conical intersection region in this archetypal Woodward-Hoffman molecule, and reflects the importance of the ionisation potentials in the analysis of photoelectron spectra. Ultimately, however, quantitative analysis requires knowledge of the photoionisation cross sections.

We proceed to investigate the utility of a range of probe photon energies in time-resolved photoelectron spectroscopy using our model, from selective low-energy $2 \mathrm{eV}$ probes to universal highenergy $15 \mathrm{eV}$ probes. In $\mathrm{CHD}$, the $2 \mathrm{eV}$ photons are tuned to probe the transient $S_{2}$ population, providing detailed insight into the dynamics. In contrast, the high-energy 9.2 and $15 \mathrm{eV}$ probes are capable of following the wavepacket all the way down to the electronic $\mathrm{S}_{0}$ ground state, providing a complete view of internal conversion and the reaction path. Interestingly, differentiation of the two products, CHD and HT, requires the highest energy $15 \mathrm{eV}$ probe since the molecules only appear different in the spectra once the excited states of the ion are considered, in agreement with previous findings. ${ }^{79,81}$ 
The calculations demonstrate that with a sufficiently highenergy probe, a complete picture of the photochemical processes can be obtained. This is particularly evident in dynamically simple systems fully described by a single pathway. However, as soon as several pathways are present, the signal becomes convoluted, making analysis complicated. If anything, including more trajectories in our model and compensating for the tendency towards overcoherence in Ehrenfest dynamics ${ }^{63}$ should strengthen this conclusion. This will be common for all universal probes where their global nature implies that selectivity is reduced. CHD is a particularly challenging example as the structural similarity of the products and starting molecule combined with similar timescales for all associated reaction paths increases the spectral congestion and hides many of the features when the data is energy-resolved only. It is clear from our analysis that it is difficult to disentangle the individual contributions to the total signal, a similar conclusion to the one drawn in a recent paper by Ruckenbauer et al. ${ }^{84}$ This strengthens the argument in favour of combining data from complementary experimental techniques, ${ }^{59}$ which can constrain calculations and inform reduced dimensionality models.

The issues associated with overlapping features and spectral congestion are also evident in other examples of global probes such as those based on diffraction ${ }^{45}$ or NEXAFS, ${ }^{58}$ with both studies using theoretically calculated dynamics to deconvolute and interpret the data. These examples, and the results in this article, support the case for accurate simulations of dynamics, but also demonstrate the need for accurate predictions of how the dynamics projects onto the measurement. With photoelectron spectroscopy specifically, there are additional experimental routes to deconvolute the contributions from the various reaction paths. This includes multiplexing the data through the collection of photoelectron angular distributions, or coincidence measurements where different products, for instance in dissociation, are produced. To extend the applicability of these situation, key steps will include extending the simulations to provide angular distributions and a more accurate description of the ionisation process such that channel interactions and the vibrational states of cation are taken into account. Measurement of ionisation via intermediate resonances may also carry extra information, but at the cost of increased complexity of the analysis. ${ }^{85,86}$ The final conclusion is that without extra information beyond the photoelectron spectrum, be that theory or angular distributions or something else, extracting information on complex and competing dynamic processes is quite challenging.

\section{Conflicts of interest}

There are no conflicts to declare.

\section{Acknowledgements}

Kenichiro Saita (Hokkaido University) is thanked for his work on the quantum molecular dynamics simulations and Samer Gozem (Georgia State University) is thanked for his help with the ezDyson calculations. RSM, AK, and MT thank the Leverhulme Trust for research funding (RPG-2013-365). AK acknowledges funding from the European Union (FP7-PEOPLE-2013-CIG-NEWLIGHT) and the Carnegie Trust via a Collaborative Research Grant held with Martin Paterson (HW). RSM acknowledges a Royal Society University Research Fellowship (UF150655). Part of the computational work reported used the ARCHER UK National Supercomputing Service (http://www.archer.ac.uk) and the Edinburgh Computer and Data Facility (ECDF) (http://www.ecdf.ed.ac.uk).

\section{References}

1 E. Havinga, R. J. D. Kock and M. P. Rappoldt, Tetrahedron, 1960, 11, 276-284.

2 E. Havinga and J. L. M. A. Schlatmann, Tetrahedron, 1961, 16, 146-152.

3 N. A. Anderson, J. J. Shiang and R. J. Sension, J. Phys. Chem. A, 1999, 103, 10730-10736.

4 Photochromism: Molecules and Systems, ed. H. Dürr and H. Bouas-Laurent, Elsevier Science, 1st edn, 2003.

5 M. Irie, Chem. Rev., 2000, 100, 1685-1716.

6 M. Irie, S. Kobatake and M. Horichi, Science, 2001, 291, 1769-1772.

7 D. Geppert, L. Seyfarth and R. de Vivie-Riedle, Appl. Phys. B: Lasers Opt., 2004, 79, 987-992.

8 S. Kobatake, S. Takami, H. Muto, T. Ishikawa and M. Irie, Nature, 2007, 446, 778-781.

9 S. Deb and P. M. Weber, Annu. Rev. Phys. Chem., 2011, 62, 19.

10 B. C. Arruda and R. J. Sension, Phys. Chem. Chem. Phys., 2014, 16, 4439-4455.

11 M. N. R. Ashfold, M. Bain, C. S. Hansen, R. A. Ingle, T. N. V. Karsili, B. Marchetti and D. Murdock, J. Phys. Chem. Lett., 2017, 8, 3440-3451.

12 N. A. Anderson, S. H. Pullen, L. A. Walker II, J. J. Shiang and R. J. Sension, J. Phys. Chem. A, 1998, 102, 10588-10598.

13 R. B. Woodward and R. Hoffmann, Angew. Chem., Int. Ed., 1969, 8, 781-853.

14 W. T. A. M. V. der Lugt and L. J. Oosterhoff, J. Am. Chem. Soc., 1969, 91, 6042-6049.

15 M. Klessinger and J. Michl, Excited States and Photochemistry of Organic Molecules, Wiley-VCH, 1st edn, 1995.

16 M. Garavelli, C. S. Page, P. Celani, M. Olivucci, W. E. Schmid, S. A. Trushin and W. Fuss, J. Phys. Chem. A, 2001, 105, 4458-4469.

17 P. J. Reid, S. J. Doig, S. D. Wickham and R. A. Mathies, J. Am. Chem. Soc., 1993, 115, 4754-4763.

18 P. J. Reid, M. K. Lawless, S. D. Wickham and R. A. Mathies, J. Phys. Chem., 1994, 98, 5597-5606.

19 M. K. Lawless, S. D. Wickham and R. A. Mathies, Acc. Chem. Res., 1995, 28, 493-502.

20 S. H. Pullen, N. A. Anderson, L. A. Walker II and R. J. Sension, J. Chem. Phys., 1998, 108, 556-563.

21 S. Lochbrunner, W. Fuss, W. E. Schmid and K.-L. Kompa, J. Phys. Chem. A, 1998, 102, 9334-9344.

22 S. Adachi, M. Sato and T. Suzuki, J. Phys. Chem. Lett., 2015, 6, 343-346. 
23 O. Schalk, T. Geng, T. Thompson, N. Baluyot, R. D. Thomas, E. Tapavicza and T. Hansson, J. Phys. Chem. A, 2016, 120, 2320-2329.

24 N. Kuthirummal, F. M. Rudakov, C. L. Evans and P. M. Weber, J. Chem. Phys., 2006, 125, 133307.

25 S. A. Trushin, W. Fuß, T. Schikarski, W. E. Schmid and K. L. Kompa, J. Chem. Phys., 1997, 106, 9386-9389.

26 W. Fuß, W. E. Schmid and S. A. Trushin, J. Chem. Phys., 2000, 112, 8347-8362.

27 K. Kosma, S. A. Trushin, W. Fuß and W. E. Schmid, Phys. Chem. Chem. Phys., 2009, 11, 172-181.

28 P. Celani, S. Ottani, M. Olivucci, F. Bernardi and M. A. Robb, J. Am. Chem. Soc., 1994, 116, 10141-10151.

29 M. Merchán, L. Serrano-András, L. S. Slater, B. O. Roos, R. McDiarmid and X. Xing, J. Phys. Chem. A, 1999, 103, 5468-5476.

30 S. Sakai and S. Takane, J. Phys. Chem. A, 1999, 103, 2878-2882.

31 H. Tamura, S. Nanbu, H. Nakamura and T. Ishida, Chem. Phys. Lett., 2005, 401, 487-491.

32 T. Mori and S. Kato, Chem. Phys. Lett., 2009, 476, 97-100.

33 A. Nenov, P. Kölle, M. A. Robb and R. de Vivie-Riedle, J. Org. Chem., 2010, 75, 123-129.

34 M. Garavelli, P. Celani, M. Fato, M. J. Bearpark, B. R. Smith, M. Olivucci and M. A. Robb, J. Phys. Chem. A, 1997, 101, 2023-2032.

35 A. Hofmann and R. de Vivie-Riedle, J. Chem. Phys., 2000, 112, 5054-5059.

36 C. Nonnenberg, S. Grimm and I. Frank, J. Chem. Phys., 2003, 119, 11585-11590.

37 H. Tamura, S. Nanbu, T. Ishida and H. Nakamura, J. Chem. Phys., 2006, 124, 084313.

38 A. Li, S. Yuan, Y. Dou, Y. Wang and Z. Wen, Chem. Phys. Lett., 2009, 478, 28-32.

39 J. B. Schönborn, J. Sielk and B. Hartke, J. Phys. Chem. A, 2010, 114, 4036.

40 A. Ohta, O. Kobayashi, S. O. Danielache and S. Nanbu, Chem. Phys., 2015, 459, 45-53.

41 J. Kim, H. Tao, T. J. Martinez and P. Bucksbaum, J. Phys. B: At., Mol. Opt. Phys., 2015, 48, 164003.

42 Y. Lei, H. Wu, X. Zheng, G. Zhai and C. Zhu, J. Photochem. Photobiol., A, 2016, 317, 39-49.

43 J. Kim, H. Tao, J. L. White, V. S. Petrovič, T. J. Martinez and P. H. Bucksbaum, J. Phys. Chem. A, 2012, 116, 2758-2763.

44 M. P. Minitti, J. M. Budarz, A. Kirrander, J. Robinson, T. J. Lane, D. Ratner, K. Saita, T. Northey, B. Stankus, V. Cofer-Shabica, J. Hastings and P. M. Weber, Faraday Discuss., 2014, 171, 81.

45 M. P. Minitti, J. M. Budarz, A. Kirrander, J. S. Robinson, D. Ratner, T. J. Lane, D. Zhu, J. M. Glownia, M. Kozina, H. T. Lemke, M. Sikorski, Y. Feng, S. Nelson, K. Saita, B. Stankus, T. Northey, J. B. Hastings and P. M. Weber, Phys. Rev. Lett., 2015, 114, 255501.

46 R. Iikubo, T. Sekikawa, Y. Harabuchi and T. Taketsugu, Faraday Discuss., 2016, 194, 147-160.

47 A. J. Orr-Ewing, J. R. R. Verlet, T. J. Penfold, R. S. Minns, M. P. Minitti, T. I. Solling, O. Schalk, M. Kowalewski, J. P. Marangos, M. A. Robb, A. S. Johnson, H. J. Wörner,
D. V. Shalashilin, R. J. D. Miller, W. Domcke, K. Ueda, P. M. Weber, R. Cireasa, M. Vacher, G. M. Roberts, P. Decleva, F. Bencivenga, D. M. Neumark, O. Gessner, A. Stolow, P. K. Mishra, I. Polyak, K. K. Baeck, A. Kirrander, D. Dowek, Á. Jiménez-Galán, F. Martín, S. Mukamel, T. Sekikawa, M. F. Gelin, D. Townsend, D. V. Makhov and S. P. Neville, Faraday Discuss., 2016, 194, 209-257.

48 E. C. Carroll, B. J. Pearson, A. C. Florean, P. H. Bucksbaum and R. J. Sension, J. Chem. Phys., 2006, 124, 114506.

49 E. C. Carroll, J. L. White, A. C. Florean, P. H. Bucksbaum and R. J. Sension, J. Phys. Chem. A, 2008, 112, 6811-6822.

50 D. Geppert and R. de Vivie-Riedle, Chem. Phys. Lett., 2005, 404, 289-295.

51 H. Tamura, S. Nanbu, T. Ishida and H. Nakamura, J. Chem. Phys., 2006, 125, 034307.

52 R. S. Minns and A. Kirrander, Faraday Discuss., 2016, 194, 11-13. 53 R. C. Dudek and P. M. Weber, J. Phys. Chem. A, 2001, 105, 4167-4171.

54 C.-Y. Ruan, V. A. Lobastov, R. Srinivasan, B. M. Goodson, H. Ihee and A. H. Zewail, Proc. Natl. Acad. Sci. U. S. A., 2001, 98, 7117.

55 H. Ihee, V. A. Lobastov, U. M. Gomez, B. M. Goodson, R. Srinivasan, C.-Y. Ruan and A. H. Zewail, Science, 2001, 291, 458.

56 J. D. Cardoza, R. C. Dudek, R. J. Mawhorter and P. M. Weber, Chem. Phys., 2004, 299, 307-312.

57 V. S. Petrovič, M. Siano, J. L. White, N. Berrah, C. Bostedt, J. D. Bozek, D. Broege, M. Chalfin, R. N. Coffee, J. Cryan, L. Fang, J. P. Farrell, L. J. Frasinski, J. M. Glownia, M. Gühr, M. Hoener, D. M. P. Holland, J. Kim, J. P. Marangos, T. Martinez, B. K. McFarland, R. S. Minns, S. Miyabe, S. Schorb, R. J. Sension, L. S. Spector, R. Squibb, H. Tao, J. G. Underwood and P. H. Bucksbaum, Phys. Rev. Lett., 2012, 108, 253006.

58 A. R. Attar, A. Bhattacherjee, C. D. Pemmaraju, K. Schnorr, K. D. Closser, D. Prendergast and S. R. Leone, Science, 2017, 356, 54-59.

59 C. C. Pemberton, Y. Zhang, K. Saita, A. Kirrander and P. M. Weber, J. Phys. Chem. A, 2015, 119, 8832.

60 A. Kirrander, K. Saita and D. V. Shalashilin, J. Chem. Theory Comput., 2016, 12, 957-967.

61 S. Gozem, A. O. Gunina, T. Ichino, D. L. Osborn, J. F. Stanton and A. I. Krylov, J. Phys. Chem. Lett., 2015, 6, 4532-4540.

62 D. V. Shalashilin, Faraday Discuss., 2011, 153, 105.

63 D. V. Makhov, W. J. Glover, T. J. Martinez and D. V. Shalashilin, J. Chem. Phys., 2014, 141, 054110.

64 F. Fabre, G. Petite, P. Agostini and M. Clement, J. Phys. B: At. Mol. Phys., 1982, 15, 1353-1369.

65 G. Petite, F. Fabre, P. Agostini, M. Crance and M. Aymar, Phys. Rev. A: At., Mol., Opt. Phys., 1984, 29, 2677-2689.

66 U. Fano and A. R. P. Rau, Atomic collisions and spectra, Academic Press, 1st edn, 1986.

67 A. Kirrander, H. H. Fielding and C. Jungen, J. Chem. Phys., 2007, 127, 164301.

68 A. Kirrander, H. H. Fielding and C. Jungen, J. Chem. Phys., 2010, 132, 024313.

69 A. Kirrander, Ch. Jungen and H. H. Fielding, Phys. Chem. Chem. Phys., 2010, 12, 8948. 
70 C. M. Oana and A. I. Krylov, J. Chem. Phys., 2007, 127, 234106. 71 C. M. Oana and A. I. Krylov, J. Chem. Phys., 2009, 131, 124114. 72 K. Saita and D. V. Shalashilin, J. Chem. Phys., 2012, 137, 22 A506.

73 H.-J. Werner, P. J. Knowles, G. Knizia, F. R. Manby and M. Schütz, et al., MOLPRO, version 2012.1, a package of ab initio programs.

74 H. Tao, PhD thesis, Stanford University, California, 2011.

75 R. C. Brown and E. J. Heller, J. Chem. Phys., 1981, 75, 186.

76 N. Zotev and A. Kirrander, Manuscript in preparation.

77 Y. Shao, L. F. Molnar, Y. Jung, J. Kussmann, C. Ochsenfeld, S. T. Brown, A. T. Gilbert, L. V. Slipchenko, S. V. Levchenko, D. P. O’Neill, R. A. DiStasio Jr, R. C. Lochan, T. Wang, G. J. Beran, N. A. Besley, J. M. Herbert, C. Yeh Lin, T. Van Voorhis, S. Hung Chien, A. Sodt, R. P. Steele, V. A. Rassolov, P. E. Maslen, P. P. Korambath, R. D. Adamson, B. Austin, J. Baker, E. F. C. Byrd, H. Dachsel, R. J. Doerksen, A. Dreuw, B. D. Dunietz, A. D. Dutoi, T. R. Furlani, S. R. Gwaltney, A. Heyden, S. Hirata, C.-P. Hsu, G. Kedziora, R. Z. Khalliulin, P. Klunzinger, A. M. Lee, M. S. Lee, W. Liang, I. Lotan, N. Nair, B. Peters, E. I. Proynov, P. A. Pieniazek, Y. Min Rhee, J. Ritchie, E. Rosta, C. David Sherrill, A. C. Simmonett, J. E. Subotnik, H. Lee Woodcock III, W. Zhang, A. T. Bell, A. K. Chakraborty, D. M. Chipman, F. J. Keil, A. Warshel, W. J. Hehre, H. F. Schaefer III, J. Kong, A. I. Krylov, P. M. W. Gill and M. Head-Gordon, Phys. Chem. Chem. Phys., 2006, 8, 3172-3191.
78 J. Wang, B. Yang, T. A. Cool, N. Hansen and T. Kasper, Int. J. Mass Spectrom., 2008, 269, 210-220.

79 K. Kimura, Handbook of HeI Photoelectron Spectra of Fundamental Organic Molecules, Japan Scientific Societies Press, Tokyo, Japan, 1981.

80 T. Horio, R. Spesyvtsev, Y. Furumido and T. Suzuki, J. Chem. Phys., 2017, 147, 013932.

81 J. Nishitani, C. W. West, C. Higashimura and T. Suzuki, Chem. Phys. Lett., 2017, 684, 397-401.

82 R. J. Squibb, M. Sapunar, A. Ponzi, R. Richter, A. Kivimäki, O. Plekan, P. Finetti, N. Sisourat, V. Zhaunerchyk, T. Marchenko, L. Journel, R. Guillemin, R. Cucini, M. Coreno, C. Grazioli, M. D. Fraia, C. Callegari, K. C. Prince, P. Decleva, M. Simon, J. H. D. Eland, N. Doslič, R. Feifel and M. N. Piancastelli, Nat. Commun., 2018, 9, 63.

83 A. D. Smith, E. M. Warne, D. Bellshaw, D. A. Horke, M. Tudorovskya, E. Springate, A. J. H. Jones, C. Cacho, R. T. Chapman, A. Kirrander and R. S. Minns, Phys. Rev. Lett., 2018, 120, 183003.

84 M. Ruckenbauer, S. Mai, P. Marquetand and L. González, Sci. Rep., 2016, 6, 35522.

85 B. Stankus, J. M. Budarz, A. Kirrander, D. Rogers, J. Robinson, T. J. Lane, D. Ratner, J. Hastings, M. P. Minitti and P. M. Weber, Faraday Discuss., 2016, 194, 525-536.

86 B. Stankus, N. Zotev, D. M. Rogers, Y. Gao, A. Odate, A. Kirrander and P. M. Weber, J. Chem. Phys., 2018, 148, 194306. 